\title{
Leakage Detection In Pipeline Using Wavelength
}

\author{
M.F Ghazali ${ }^{1}$, A. K Samta ${ }^{1}$ \\ ${ }^{1}$ Faculty of Mechanical Engineering, Universiti Malaysia Pahang, Malaysia \\ Email:fairusham@ump.edu.my
}

\begin{abstract}
Nowadays natural gas transport and distribution is a complex and currently growing and increasing. Natural gas produced from well need to transport in a great distance before reaching it point of use. The pipeline is designed to quickly and efficiently transport the gas from its origin to the high demand area. Either pipelines transportation for water supply or natural gas, leakage is unacceptable problem. Small leak along the pipeline is hard to detect. The objective of this study is to build the test rig galvanized iron and MDPE pipelines. Besides that, the main objective is to determine the leak detection in gas pipeline using wavelet-based filtering. . Main point of each journal is compared in order to determine the problems arise from the previous research. It is then follow by the methodology which will discuss further in this chapter. From methodology, it is known that the data taken can be analysing through Daisy Lab and Math lab software Wavelet and cross correlation is used to analyse the signal in Matlab. From the result, it show that the leak can be identified based on the peak of amplitude of the signal. The result for galvanized iron pipe is not acceptable due to short pipeline length. Thus it can be concluded that leak can be determined using wavelet-based filtering. As the conclusions, the propose technique can be used to determine the leak in pipelines
\end{abstract}

Keywords Leakage, Wavelength, Wavelet

Paper type research paper

\section{INTRODUCTION}

The worldwide natural gas transport and distribution is a complex and currently growing and increasing. Natural gas produced from well need to transport in a great distance before reaching it point of use. The pipeline is designed to quickly and efficiently transport the gas from its origin to the high demand area. Even though pipelines transportation is safe, it doesn't mean that it is out of harm[1]. Pipelines is an efficient and economic transportation however, the risks associates with accidental releases of transported product are still high [2]. Due to many reasons, they are many problem arise when this process occur. In the British companies, around 20 to $40 \%$ of all the treated water gets lost through leakage before arriving at consumers. Either pipelines transportation for water supply or natural gas, leakage is unacceptable problem. Small leak along the pipeline is hard to detect. When the leak has grown bigger and cause major problem, it already too late to control. Leak can be cause by the age factor of the pipe, excess sour gas pass through, low maintenance management and many more. Leakage is a costly problem, thus is should be prevent with much effort. It not only wastes the precious natural resource but also in economic term. Besides that, leaky pipe creates a public health risk either for water transportation or natural gas. Furthermore, leak can also cause environmental impact. There are four main categories of pipe line failures. These are pipeline corrosion and wear, operation outside design limits, unintentional third party damage and intentional damage. This problem can cause major lost to the company. In order to determine the location of the leak and size of the leak, most techniques have been invented. The most basic method of leak detection is walking, driving or flying the pipeline right away to look for evidence and discoloration of vegetation near the line or actually hear or see the leak [3]. However, most of the basic method is time consuming. The project is basically related to pipe leakage detection. The objectives of this project are to build the galvanic pipe and MDPE test rig for leak detection, to determine leak detection in gas pipelines using wavelet-based filtering and to identify the location of the leak.

Leak detection techniques have been introduced during the last decades to counteract the disastrous effects of gas leaks. Since at least one reportable leak incident reported each year from 2001 to 2005 period [4]. Therefore, leak detection techniques are considered important in order to prevent the incident from happen. However, gas leak itself is not sufficient to determine which leak 
detection technique need to be used. Size of the leak and its location need to be identify in order to determine which techniques to use.

Leak detection techniques can be classified into three categories [5]. Classification of each technique based on several criteria such as amount of human involvement needed, the physical quantity measured and the technical nature of the methods. However, the most common way to classify leak detection technique is based on their technical nature. Thus, two main categories can be distinguished which is hardware based method, and software based method. Figure one show the categories. Hardware based method is mainly based on the usage of specific sensing devices in order to detect the leak. Based on the type of sensor used and its equipment, it can be further classify into acoustic, cable sensor, vapour sampling, optical, soil monitoring and ultrasonic flow meter. Meanwhile, for software based method, it related to the usage of software programs at their core. Based on the approaches of each methods, it can be further classify into mass/volume balance, negative pressure wave, digital signal processing, real time transient modelling, pressure point analysis and statistical.

\section{Wavelet}

Wavelet is a multi resolution analysis which analyse signal at different frequencies with different resolution. It also known that wavelet transform decomposes a signal into variety of different waves, centred around different frequencies [6]. Wavelet means small wave which smallness refers to state of the window function is of finite length meanwhile wave refers to the state that this function is oscillatory. It works by giving good time resolution and poor frequency resolution at high frequencies and vice versa. Wavelet transform evolved from Fourier transform, the signal is multiple by wavelet function. It involves the same way as complex expansion which include sine and cosine functions.

Continuous wavelet transform is however different from discrete wavelet transform. Continuous wavelet transform provides information that is highly redundant and require considerable amount of computation time and resources meanwhile discrete wavelet transform provides enough information for both analysis and synthesis of the original signal. Theoretically, wavelet can be calculated as follow;

$$
Q(a, b)=\frac{1}{\sqrt{a}} \int_{-\infty}^{\infty} y(t) \varphi^{*}\left(\frac{t-b}{a}\right) d t
$$

Where Q ( $\mathrm{a}, \mathrm{b})$ is continuous wavelet transform. This signal consists of a function with two variables which a stand for translation and $\mathrm{b}$ stand for scale parameter. Meanwhile $\varphi^{*}$ stand for complex conjugate of the basic function. It is also known as mother wavelet. Mother wavelet represents two important properties of wavelet analysis. Wavelet means small wave where smallness refers to the condition that the function is of finite length which means that it is compactly supported [7]. Wave itself refers to the condition that this function is oscillatory. Furthermore, the term mother means that the derivation of different region of function in the transformation process is based on one main function thus mother wavelet means a prototype of generating window function[8].

\section{METHOD}

Methodology is a chapter where all procedure, method and analysis are known in order to obtain a result for the experiment. Besides that, material selection is also known. Project flow is discussed in order to identify the work flow and schedule. It starts with the discussion of the topic and the main objectives of this study. A limitation of the experiment is discussed under the scope topic. Background study of the topic is known in order to determine the main objectives of the projects. Background study is done through the reading of previous related journal and paper. Main point of each journal is compared in order to determine the problems arise from the previous research. It is then follow by the methodology which will discuss further in this chapter. From methodology, it is known that the data taken can be analysing through Daisy Lab and Math lab software. After the result is validated and analyse, any error that occur will lead to the repetition of the experiment and analysis. It is done until good result is obtained.

Second flow chart is the experiment flow chart. Experiment flow start with designing the stand for the pipelines. A support is needed for the test rig to stand on its own. Material selection of the pipe 
is known with software and experimental setup. Among the material to select is type of pipe used, microphone, and compressor. NI-DAQ Max and daisy lab is used in software setup meanwhile test rig is being prepared for experimental setup. Data collection is measured based on the flow of air throughout the experiment. Data is then being analyse using math lab software. Any error in the data collection will lead to the repetition of the experiment.

Methods use in leak detection is based on echoes reflected from turbulence induced by a leak. They are many methods of leak detection which mainly based on process variables such as flow rate, pressure and temperature. Previously, leak has been detected using 'listening rod' where a rod that is made up of simple amplifiers detects the sound induced by water leaking from pressurized pipe. However, this method is not suitable for gas pipelines since gas pipelines are long. After a few years, the improvements have been made for leak detection method. Ground microphone and correlators are invented to improve the speed and accuracy of leakage surveys. Besides, for sensitive mechanisms or materials, electronic listening devices are invented such as piezoelectric. Piezoelectric function as a sense to leak induced sound or vibration improved on the range and accuracy of listening rod. This study shows an improvement method of leak detection. A microphone act as sense to induced sound. A compressor is used in this study as the main source of the background noise.

Medium density polyethylene (MDPE) pipe is one of the commonly used pipes in gas pipes and fittings, carrier bags and screw closure. It is thermoplastic type of polymer. MDPE has better stress cracking resistance than High Density Polyethylene (HDPE). the material and mechanical properties of the MDPE used in this experiment.

Microphone used in the project is a pre-polarized (OV) condenser microphones amplifier. It is widely used in acoustic project. It consists of microphone cartridge, a mated preamplifier with TEDS and a system calibration. Speaker used in the project is a multimedia speaker system. It is a portable speaker which using latest ABS engineering plastic material. It uses USB interface and full support windows operating system.

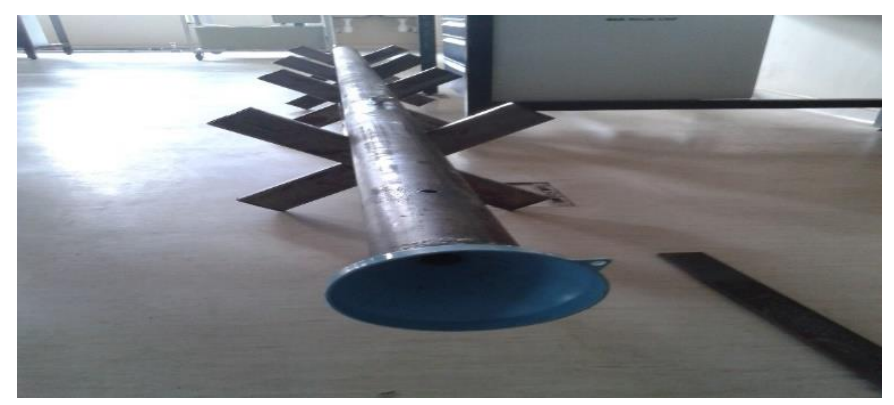

Figure 1 Actual test rig

\section{DISCUSSION}

Microphone detected the reflected signal from the original signal. The data from the original signal can be constructed as follow; 


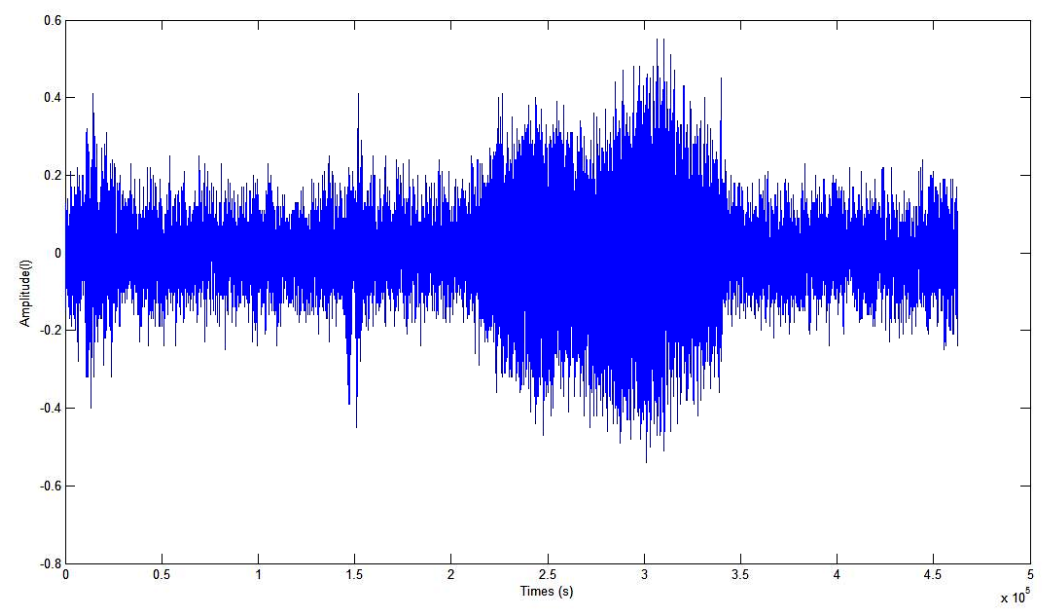

Figure 2 Raw data of signal

Based on figure 2, the data shown do not indicate whether there is leak or not in the piping system. Therefore, a thorough analysis need to be done for the signal acquire. When Matlab analysis is applied to the raw signal, it will filter the noise. Input signal for this project include chirp signal with $3.5 \mathrm{~Hz}$ frequency. Chirp signal also includes the block size of 2000 and sampling rate of 51.2k Hz. Besides that, to indicate whether leak is detected or not in pipelines, a significant echo will reflect in the construct signal. Furthermore, the highest amplitude can also indicate that area containing leak or not. Inverse continuous wavelet transform will determine the reflected echo based on the highest kurtosis value calculated. Wavelet analysis is among many ways to analyse the signal. When the signal is collected, it is imported to wavelet toolbox for one dimensional analysis. Continuous wavelet transform is chosen to analyse the signal. Two conditions are applied during data acquisition which includes; Pipe containing leak and Pipe without leak.

However, by just observing raw data, the result cannot be concluded. Therefore, the comparison between two waves will determine which one is the leak condition pipeline or not. Both conditions is apply for both types of pipe used. Figures below will show the data after wavelet based filter, which used Mexican hat as mother wavelet. The coefficients from continuous wavelet transform with scale of 90, is then used to determine the kurtosis value for each conditions. Kurtosis value for each scale is calculated to determine the highest value of the kurtosis. The highest value of kurtosis is analysed using inverse wavelet transform.

\section{Pipe without leak}

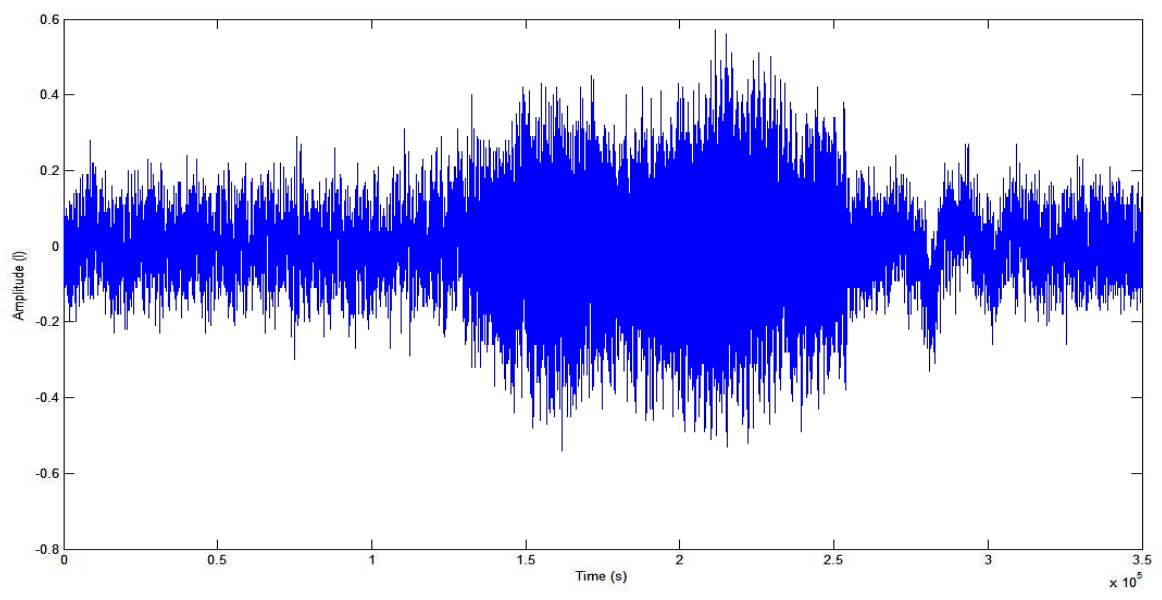

Figure 3 Raw data of signal without leak 


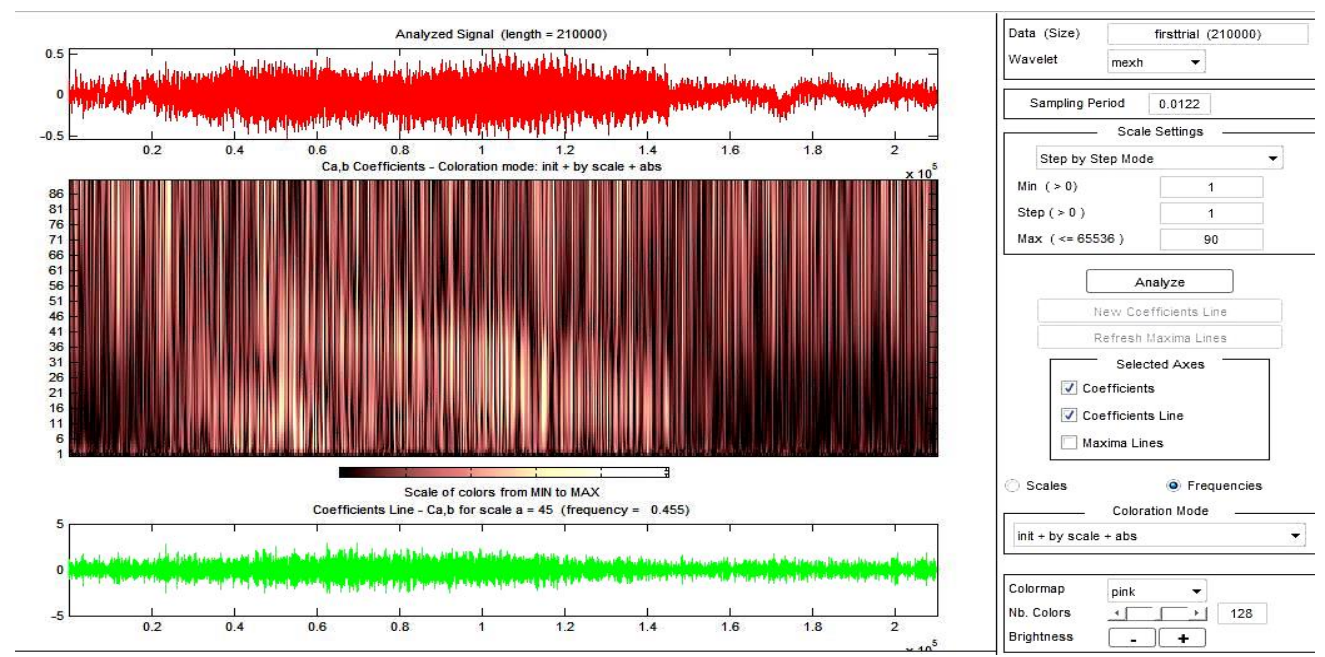

Figure 4 Filtered signal using continuous wavelet transform
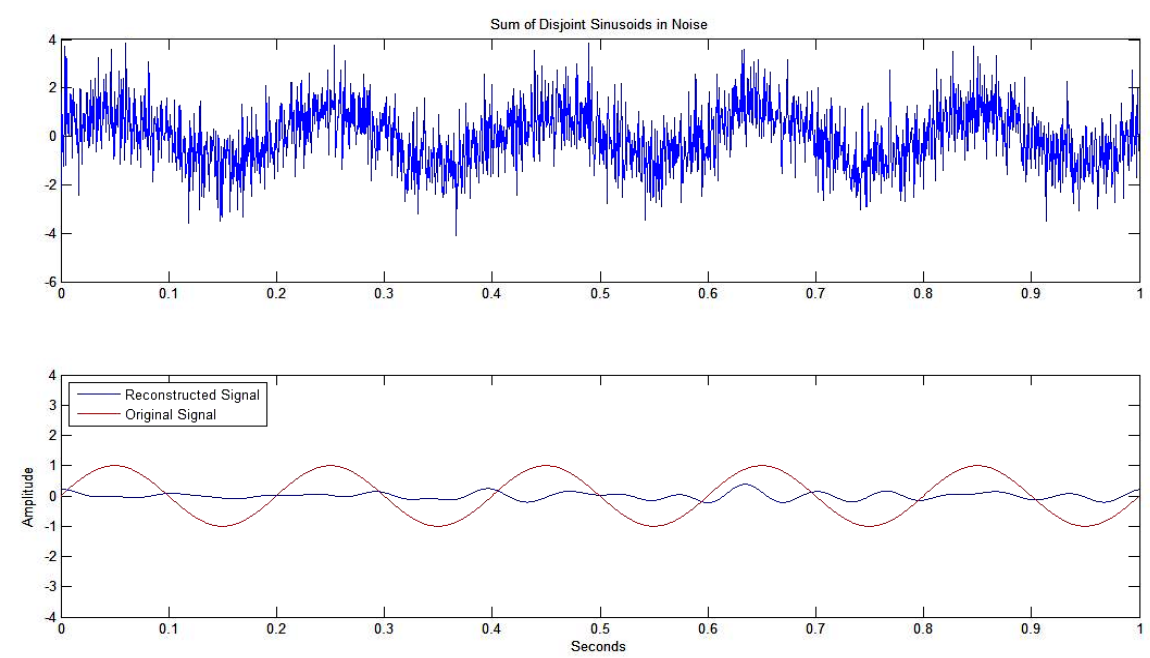

Figure 5 Inversed continuous transform for signal without leak

Based on the condition pipeline without leak, a test has been conducted to prove that the condition is true. Chirp signal with frequency range from $20-600 \mathrm{~Hz}$ was inserted into the pipelines. Signal is then filtered and the kurtosis value for each scale is calculated. The sampling period for filtered is $0.0122 \mathrm{~s}$ and the scale is 45 . Based on the filtered signal, a kurtosis value for each scale is calculated. The highest kurtosis value was 5.3214.Then the highest kurtosis is reconstructed to form the new sine wave. The amplitude of the end of the pipe is measured. The end amplitude of the pipe is $0.118 \mathrm{~mm}$.

\section{Pipelines with leak}




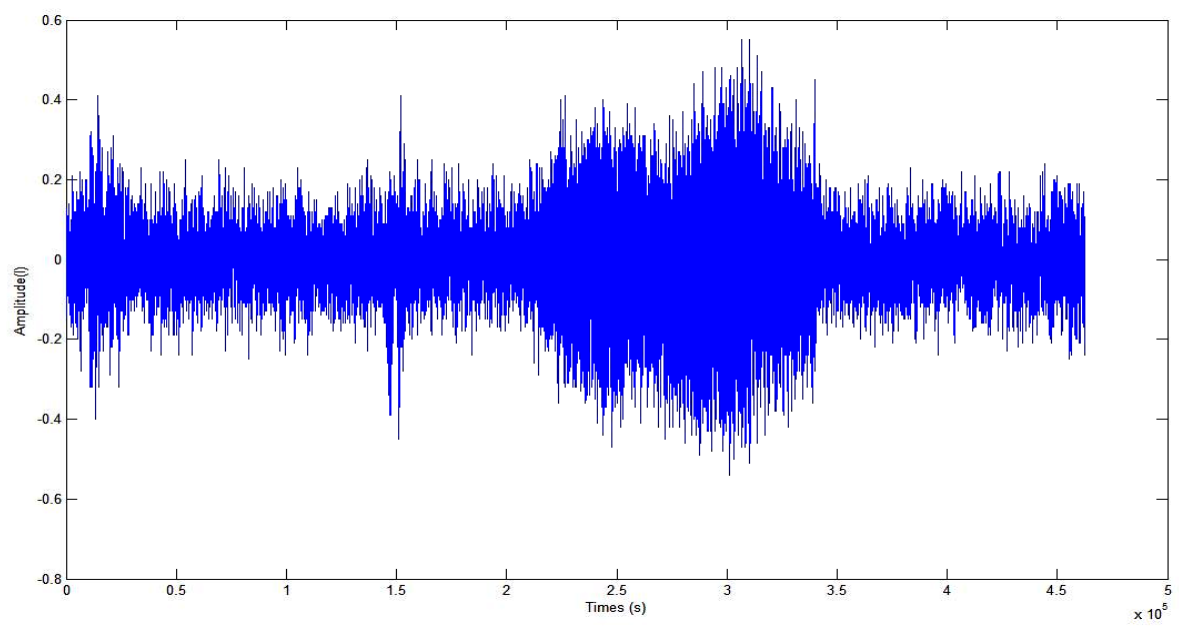

Figure 6 Raw data for leak pipeline

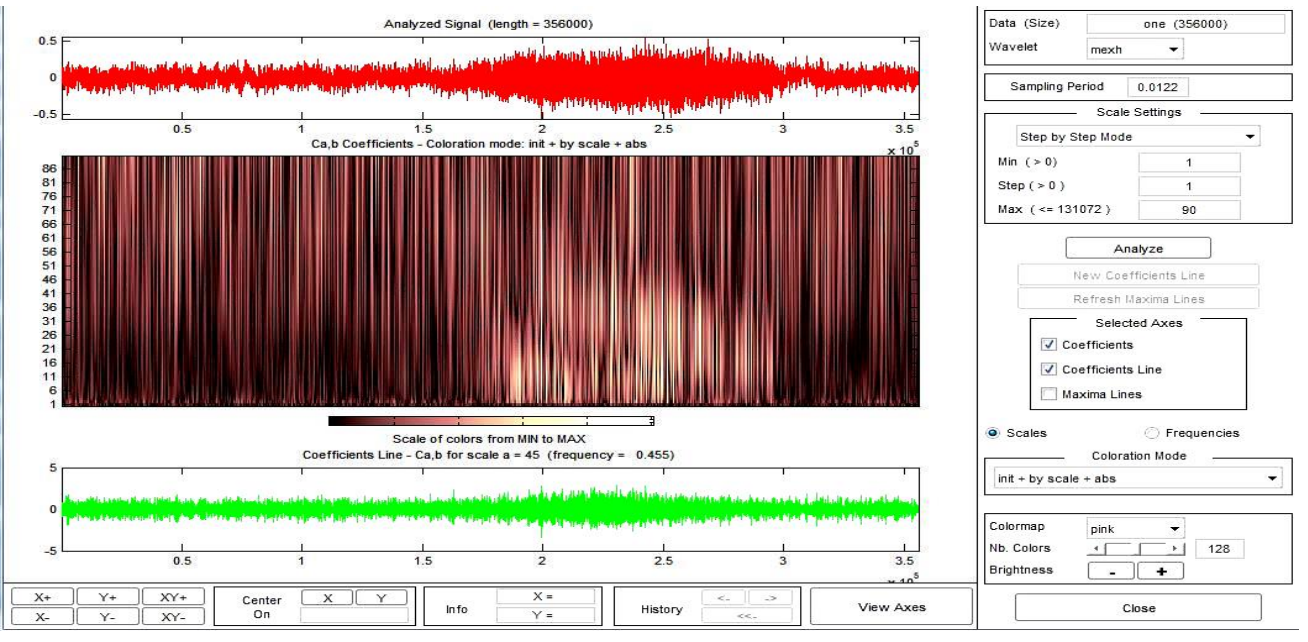

Figure 7 Sampled data and filtered using wavelet transform

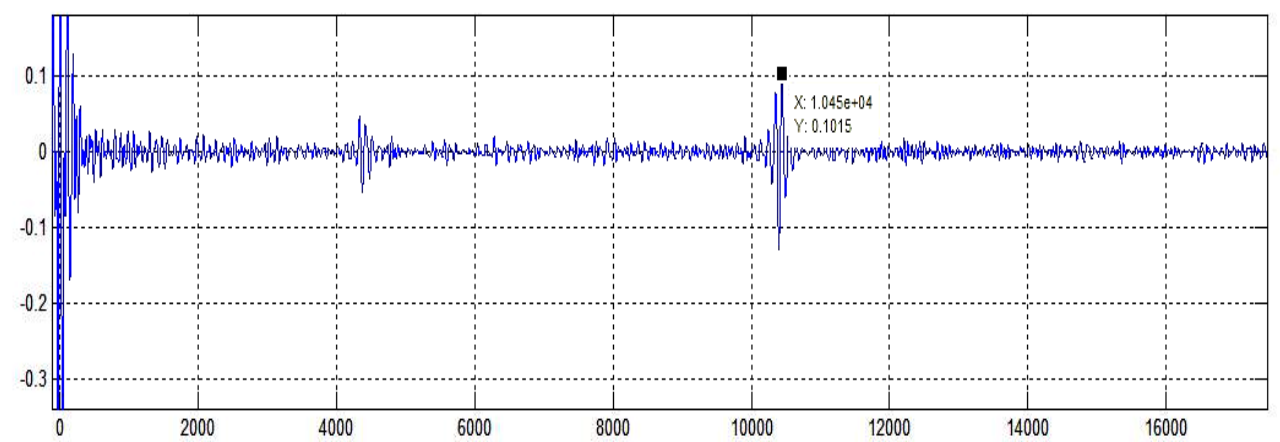

Figure 8 Inverse signal from the highest kurtosis value

For pipelines with leak, the signal is filtered using wavelet based filtering and Mexican hat. The same signal which is chirp signal was inserted into the pipelines containing leak. The signal is filtered using the Mexican hat and the sampling period is $0.0122 \mathrm{~s}$. The scale for the filtered signal is 45. From the coefficient of the filtered signal, a kurtosis value for each scale is calculated. The highest kurtosis value is 5.5575 and in a scale number 8 . The data from highest kurtosis value is then transposed and inverse using continuous wavelet transform. The amplitude for hole and end of the 
pipe was measured. The home amplitude is $0.03262 \mathrm{~mm}$ meanwhile the open end amplitude is 0.11 $\mathrm{mm}$.

Based on the obtained result, it shows that the open end amplitude for both leak and no leak is approximately in the same range. Based on the inverse signal, it shows that there is reflection in wave which when compared to the no leak graph, show a lot of differences. Compare both signal, the reflected wave show the difference in amplitude for leak location meanwhile there is no difference in amplitude in no leak signal.

Pipelines with leak will create turbulence hence the differences in amplitude. High turbulence will produce high echoes as seen on the figure 4-7 above. The figure show significant echoes produce where the first excitation is based on reflected echoes at leak meanwhile the second significant excitation is the open end of the pipe.

\section{CONCLUSIONS}

All the data collected using Dasylab and analyse using Matlab is obtained from the experiment conducted for leak detection in gas pipeline using wavelet-based filtering. Wavelet based filtering is one of the way by using Mexican hat as the mother wavelet. A continuous wavelet transform is one of the ways used as the filtering method for signal which can acts as band pass filter. When mother wavelet is applied to the signal, this filter extracts the frequency content occurring at a given time. Meanwhile inverse continuous wavelet transform allow signal to be carried out in wavelet domain and the signal can be reconstructed later. The objective of this study is to build the test rig galvanized iron and MDPE pipelines. Besides that, the main objective is to determine the leak detection in gas pipeline using wavelet-based filtering. Wavelet and cross correlation is used to analyse the signal in Matlab. From the result, it show that the leak can be identified based on the peak of amplitude of the signal. The result for galvanized iron pipe is not acceptable due to short pipeline length. Thus it can be concluded that leak can be determined using wavelet-based filtering. As the conclusions, the propose technique can be used to determine the leak in pipelines.

\section{References}

[1] Pal-Stefan Murvay, I. S. (2012). A survey on gas leak detection and localization techniques. Elsevier, 966-973. doi: 10.1016/j.jlp.2012.05.010

[2] Da Silva, H. V., Morooka, C. K., Guilherme, I. R., da Fonseca, T. C., \& Mendes, J. R. P. (2005). Leak detection in petroleum pipelines using a fuzzy system. Journal of Petroleum

[3] Colombo, A. F., Lee, P., \& Karney, B. W. (2009). A selective literature review of transient-based leak detection methods. Journal of Hydro-environment Research, 2(4), 212-227. doi: 10.1016/j.jher.2009.02.003

[4] Beck, S., H, H., \& RF, B. (1995). Transmission line modeling of simulated drill strings undergoing water-hammer. Journal of Mechanical Engineering Science, 209, 419-427.

[5] Ghidaoui, M. S., Zhao, M., McInnis, D. A., \& Axworthy, D. H. (2005). A Review of Water Hammer Theory and Practice. Applied Mechanics Reviews, 58(1), 49. doi:

[6] S.B.M Beck, J. F. a. W. J. S. Wavelet and Cepstrum Analysis of leaks in pipe network.

[7] Srirangarajan, S., Allen, M., Preis, A., Iqbal, M., Lim, H. B., \& Whittle, A. J. (2012). Wavelet-based Burst Event Detection and Localization in Water Distribution Systems. Journal of Signal Processing Systems, 72(1), 1-16. doi: 10.1007/s1 1265-012-0690-6

[8] Staszewski, S. B. M. B. a. W. J. Cepstrum analysis for identifying reflection points in pipeline. 